\author{
Military Technical College \\ Kobry Elkobbah, \\ Cairo, Egypt
}

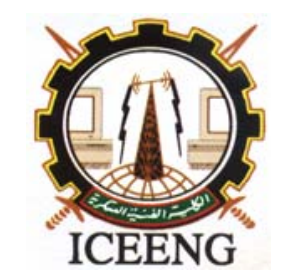

\section{$5^{\text {th }}$ International Conference on Electrical Engineering ICEENG 2006}

\title{
INVESTIGATION OF RESTORATION TECHIQUES FOR REMOTE SENSING SATELLITE IMAGERY
}

Fawzy ElTohamy Hassan ${ }^{*}$, Ph.D. Gouda Ismail", Ph.D.

\author{
M. Sharawy Ibrahim ${ }^{* *}$, Prof. Dr. Esam Hassan Hamza ${ }^{*}$,B.Sc.
}

\begin{abstract}
:
This paper introduces implementation of some image restoration techniques, which can be applied for satellite images. These techniques are, inverse filter, iterative method, wiener filter, regularized deconvolution filter and wavelet-based method. The restoration techniques are applied on several satellite images associated with atmospheric turbulence blur at different variance of additive noise to check the performance of each technique and its capability to restore the degraded image as close as possible to the original image. Also, comparison studies between these techniques are introduced based on measures like Peak Signal-to-Noise Ratio (PSNR) and Root Mean Square Error (RMSE). The experimental results show that: the wavelet method is the most suitable restoration technique for satellite images, since it gives high PSNR and small RMSE with respect to the other restoration techniques.
\end{abstract}

Keyword: Image restoration, Remote sensing, Image blur models.

\section{1- Introduction}

When image data is recorded by sensors on satellites and aircrafts. It may contain some degradations. The degradation comes in many forms such as motion blur, noise, atmospheric turbulence and camera misfocus. Thus when an image is to be utilized, it is necessary to make corrections. These corrections are achieved by image restoration, which is a process that attempts to recover an image that has been degraded by using some priori knowledge of the degradation phenomenon. Therefore restoration techniques are oriented toward modeling the degradation and applying the inverse process in order to recover the original image [1].

The image restoration techniques that are described in this paper in frequency domain and fall under the class of linear spatially invariant restoration filters. Assuming that the blurring function acts as a convolution kernel or point-spread function (PSF) that does not vary spatially. It is also assumed that the statistical properties (mean and correlation function) of the image and noise do not change spatially. Under these conditions the restoration process can be carried out by means of a linear filter of which the PSF is spatially invariant, i.e., is constant throughout the image [2].

This paper is organized as follows: Section 2 presents the problem statement and the proposed solution of the image degradation / restoration process. Section 3 introduces the atmospheric turbulence blur model. Section 4 introduces a study analysis on some of restoration techniques. Section 5 shows the analysis that is performed on the most popular restoration techniques, and the comparative study between these techniques. Section 6 concludes the work results. Finally, references are given in section 7.

* Egyptian Armed Forces 


\section{2- Problem statement}

The general problem is the restoration (deconvolution) of a satellite image from blurred and noisy data. Fig. (1) shows, the degradation model, which is represented in the spatial domain by [1], [2]:

$$
g(x, y)=d(x, y) \otimes \otimes f(x, y)+\eta(x, y)
$$

Where $f(x, y)$ and $g(x, y)$ denote respectively the original and observed degraded image, $d(x, y)$ is the impulse response of the degradation function, $\otimes \otimes$ two-dimensional (2-D) discrete linear convolution and $\eta(x, y)$ is the additive noise. Sometimes the noise is assumed to have a Gaussian probability density function with zero mean and to be white.

Fig. (1) shows the objective of restoration process is to obtain an estimate $f^{\wedge}(x, y)$ to be as close as possible to the original image $f(x, y)$ given $g(x, y)$ and $d(x, y)$. In general, the more known about $d$ and $\eta$ the $\operatorname{closer} f^{\wedge}(x, y)$ will be to $f(x, y)$.

So the equivalent frequency domain of Eq. (1) is [1], [2]:

$$
G(u, v)=D(u, v) * F(u, v)+N(u, v)
$$

Where $(u, v)$ are the spatial frequency coordinates, and capitals represent Fourier transforms.

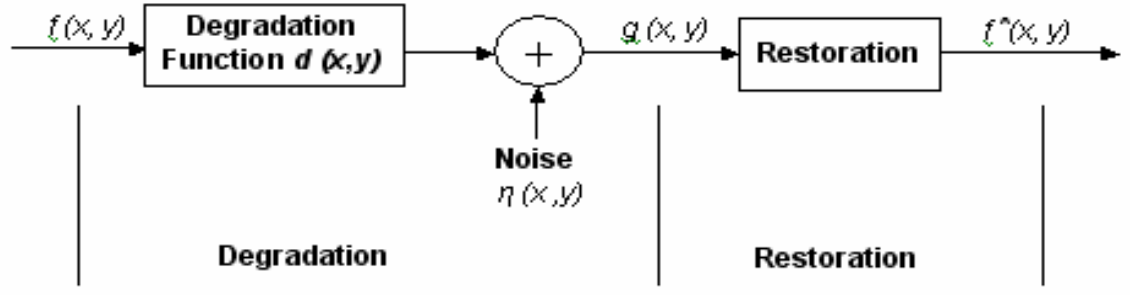

Fig. 1. A model of the image degradation / restoration process

\section{3- Atmospheric Turbulence Blur Model}

The PSF $d(x, y)$ describes how the imaging system distributes the radiation field received from a point source or point scatter and hence governs the resolution that can be obtained. The PSF also determines the frequency content of the image since high resolution is associated with high frequency information. The narrower the PSF, the greater the frequency content of the image and greater its resolution. The result of deconvolving (deblurring) an image is to increase its spatial frequency content and hence restore its resolution [3]. For spatially continuous blurs and for spatially discrete blurs the PSF is constrained to satisfy [2]:

$$
\begin{gathered}
\int_{-\infty}^{\infty} \int_{-\infty}^{\infty} d(x, y) d x d y=1 \\
\sum_{n 1=0}^{N} \sum_{n 2=0}^{-1} d\left(n_{1}, n_{2}\right)=1
\end{gathered}
$$


The common type of PSF that affect the satellite images is the atmospheric turbulence, which is a severe limitation in remote sensing. Although the blur introduced by atmospheric turbulence depends on a variety of factors (such as temperature, wind speed, and exposure time), for long - term exposures the PSF can be described reasonably well by a Gaussian function. Here $\sigma_{G}$ determines the amount of spread of the blur, and constant $\mathrm{C}$ is to be chosen so that Eq. (3) is satisfied [2].

$$
d\left(x, y ; \sigma_{G}\right)=C \exp \left(-\frac{x^{2}+y^{2}}{2 \sigma_{G}{ }^{2}}\right)
$$

\section{4- Image Restoration Techniques}

In this section the PSF of the blur is satisfactory known. The methods will be introduced for removing the blur from the recorded image using a linear filter are inverse filter, iterative method, wiener filter, regularized deconvolution filter and wavelet-based method.

\subsection{Inverse Filter}

The simplest approach to restoration is direct inverse filtering, where an estimate $F^{\wedge}(u, v)$, of the original image simply is computed by dividing the degraded image, $G(u, v)$, by the degradation function $D(u, v)$ in the absence of noise. The inverse filtering responds very badly to any noise in the image because noise tends to be high frequency [4].

$$
F \wedge(u, v)=\frac{G(u, v)}{D(u, v)}
$$

Substituting the right side of Eq. (2) for $G(u v)$ in Eq. (6) yields

$$
F \wedge(u, v)=F(u, v)+\frac{N(u, v)}{D(u, v)}
$$

In the ideal case, invert all the elements of $\mathbf{D}$ to get a high pass filter. However, notice that a lot of the elements in $\mathbf{D}$ have values either at zero or very close to it. Inverting these elements would give us either infinities or some extremely high values. In this case the ratio $N(u, v) / D(u, v)$ could easily dominate the estimate $F^{\wedge}(u, v)$. In order to avoid these values, D should be thresholding before taking the inverse, as follows [5]:

$$
D(u, v)=\left\{\begin{array}{cc}
D(u, v) \text { if } & D(u, v)>n \\
n & \text { otherwise }
\end{array}\right.
$$

Where $n$ is a threshold, and set arbitrarily close to zero for noiseless cases, but in case of noise it higher than zero.

\subsection{Iterative Method}

The idea behind the iterative procedure is to make some initial guess of $f$ based on $g$ and to update that guess after every iterations. The basic form of iterative restoration filters is the one that iteratively approaches the solution of the inverse filter, and it is given by the following spatial domain iteration:

$$
f_{o}^{\wedge}\left(n_{1}, n_{2}\right)=\lambda * g\left(n_{1}, n_{2}\right)
$$




$$
f_{k+1}^{\wedge}\left(n_{1}, n_{2}\right)=f_{k}^{\wedge}\left(n_{1}, n_{2}\right)+\lambda *\left(g\left(n_{1}, n_{2}\right)-f_{k}^{\wedge}\left(n_{1}, n_{2}\right) * * d\left(n_{1}, n_{2}\right)\right)
$$

Where $f_{o}^{\wedge}$ is an initial guess usually in the first iteration is chosen to be identical to zero or identical to $g$. Here $f_{k}^{\wedge}$ is the restoration result after $\mathrm{k}$ iterations. If the number of iterations becomes very large, then $f_{k}^{\wedge}$ approaches the solution of inverse filter. If our $f_{k}^{\wedge}$ is a good guess, eventually $f_{k}^{\wedge}$ convolved with $d$ will be close to $g$. When that happens the second term in the $f^{\wedge}{ }_{k+1}$ equation will disappear and $f_{k}^{\wedge}$ and $f^{\wedge}{ }_{k+1}$ will converge. $\lambda$ is our convergence factor and it lets us determine how fast $f_{k}^{\wedge}$ and $f^{\wedge}{ }_{k+1}$ converge [6], [7].

If we take both of the above equations to the frequency domain, we get

$$
\begin{gathered}
F_{o}^{\wedge}(u, v)=\lambda * G(u, v) \\
F_{k+1}^{\wedge}(u, v)=f_{k}^{\wedge}(u, v)+\lambda *\left(G(u, v)-F_{k}^{\wedge}(u, v) * D(u, v)\right)
\end{gathered}
$$

The convergence occurs if the convergence parameter $\lambda$ satisfies:

$$
|1-\lambda D(u, v)|<1 \text { for all }(u, v)
$$

Where $D(u, v)$ is the 2D discrete Fourier transform (DFT) of $d(x, y)$ and $|z|$ denotes the magnitude of a complex number z. Using the fact that $|D(u, v)| \leq 1$, this condition simplifies to [8]:

$$
0<\lambda<2, \quad D(u, v)>0
$$

\subsection{Minimum Mean Square Error (Wiener) Filtering}

Wiener deconvolution can be used effectively when the frequency characteristics of the original image and additive noise are known. The Wiener filtering executes an optimal tradeoff between inverse filtering and noise smoothing. It removes the additive noise and inverts the blurring simultaneously. In the absence of noise, the Wiener filter reduces to the ideal inverse filter. The Wiener filtering is optimal in terms of the mean square error. In other words, it minimizes the overall mean square error (MSE) between the ideal and restored image in the process of inverse filtering and noise smoothing [1].

$$
M S E=\mathrm{E}\left(f\left(n_{1}, n_{2}\right)-f^{\wedge}\left(n_{1}, n_{2}\right)\right)^{2}
$$

Where $\mathrm{E}\{$.$\} is the expected value of the argument. The Wiener filter in Fourier domain can$ be expressed as follows [1], [9]:

$$
W(u, v)=\frac{D^{*}(u, v)}{|D(u, v)|^{2}+\frac{S_{\eta \eta}(u, v)}{S_{x x}(u, v)}}
$$

Where $S_{x x}(u, v)=|F(u, v)|^{2}, S_{\eta \eta}(u, v)=|N(u, v)|^{2}$ are respectively power spectrum of the original image and power spectrum of additive noise, $D(u, v)$ is blurring filter and $D^{*}(u, v)$ is a complex conjugate of $D(u, v)$. It is easy to see that the wiener filter has two separate parts, an inverse filtering part and a noise smoothing part. It doesn't only perform the deconvolution by inverse filtering (high-pass filtering) but also removes the noise with a compression operation (low-pass filtering). When dealing with spectrally white noise, the spectrum $|N(u, v)|^{2}$ is a constant, which simplifies things considerably. However, the power spectrum of the undegraded image seldom is known. 
An approach used frequently when these quantities are not known or cannot be estimated is to approximate Eq. (16) by the expression [1], [2]:

$$
W(u, v)=\frac{D^{*}(u, v)}{|D(u, v)|^{2}+\frac{1}{(S N R)^{2}}}
$$

Where $S N R$ is a constant represents a Signal-to-Noise ratio. The estimated image can be obtained by:

$$
F^{\wedge}(u, v)=W(u, v) \quad G(u, v)
$$

\subsection{Constrained Least Squares Filtering (Regularized filter)}

Regularized filter is another approach for overcoming some of the difficulties of the inverse filter (excessive noise amplification) and of the Wiener filter (estimation of the power spectrum of the original image). A regularized filter requires Knowledge of only the mean and variance of the noise. These parameters usually can be calculated from a given degraded image, so this is an important advantage than the Wiener filter. A more reasonable expectation for the restored image is that it satisfies [1], [3], [9]:

$$
\sum_{n_{1}=0}^{N} \sum_{n_{1}=0}^{M}\left(g\left(n_{1}, n_{2}\right)-d\left(n_{1}, n_{2}\right) * * f^{\wedge}\left(n_{1}, n_{2}\right)\right)^{2} \approx \sigma_{\eta}^{2}
$$

Where $\sigma_{\eta}^{2}$ is noise variance. The regularized filter in Fourier domain can be expressed as follows:

$$
H_{c l s}(u, v)=\frac{D^{*}(u, v)}{|D(u, v)|^{2}+\gamma|P(u, v)|^{2}}
$$

Here $\gamma$ is a tuning or regularization parameter that should be chosen such that Eq. (19) is satisfied, and $P(u, v)$ is the Fourier transform of the PSF of a 2-D high-pass filter.We recognize this function as a 2-D Laplacian operator as follows [1], [10]:

$$
p(x, y)=\left[\begin{array}{ccc}
0 & -1 & 0 \\
-1 & 4 & -1 \\
0 & -1 & 0
\end{array}\right]
$$

The estimated image can be obtained by:

$$
F \wedge(u, v)=H_{c l s}(u, v) G(u, v)
$$

\subsection{Wavelet-Based Method}

The Wiener filtering is the optimal tradeoff of inverse filtering and noise smoothing but in the case when the blurring filter is singular, the Wiener filtering actually amplifies the noise. Thus a denoising step is needed to remove the amplified noise. Wavelet method is a successful approach introduced recently by R. Neelamani et al. The idea of wavelet-based deconvolution technique for ill-conditioned systems is to employ both Fourier-domain Wiener-like and wavelet-domain regularization. The regularized inverse filter is introduced by modifying the wiener filter with a new-introduced parameter [11], [12]:

$$
G_{\alpha}(u, v)=\frac{D^{*}(u, v) S_{x x}(u, v)}{|D(u, v)|^{2} S_{x x}(u, v)+\alpha S_{\eta \eta}(u, v)}
$$


The parameter $(\alpha=0.27)$ can be optimally selected to minimize the overall mean-square error. The block diagram of the Wavelet restoration algorithm is displayed in Fig. (2) [13].

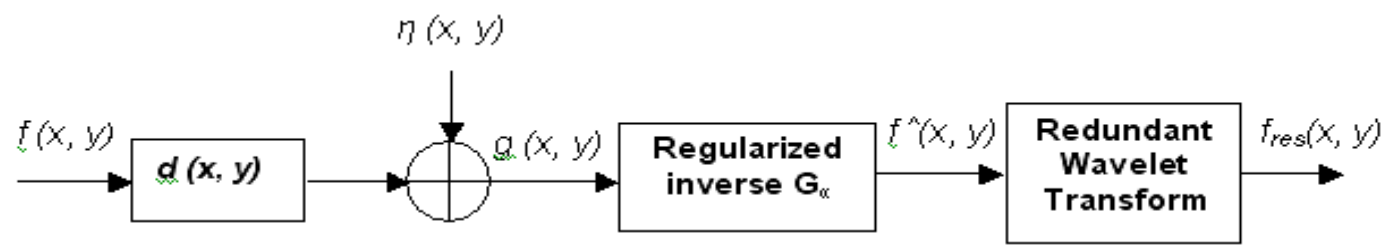

Fig. 2. The block diagram of the Wavelet restoration algorithm

The operation start by applying the regularized inverse filter in Fourier domain to cancel the blurring in the noisy degraded image $g$ to obtain $f^{\wedge}$. Apply the wavelet denosing algorithm in wavelet domain to reduce (smooth) the noise in $f^{\wedge}$ to obtain the restored image $f_{\text {res }}$.

\section{5- Experimental work}

The analysis is performed on inverse filter, iterative method, wiener filter, regularized deconvolution filter and wavelet-based method as the most popular restoration techniques. The comparative study between these techniques is applied on several satellite images with atmospheric turbulence blur at three different noise level using PSNR and RMSE as a metric measures.

To compare between these algorithms a set of 10 satellite sub-images are used namely imgsat 1, imgsat 2,...imgsat 10 respectively. The images are acquired by the commercial Remote Sensing satellites SPOT 5 (imgsat 1, imgsat 3, imgsat 5, imgsat 7, imgsat 9) and LANDSAT 7 (imgsat 2, imgsat 4, imgsat 6, imgsat 8, imgsat 10). The images format is TIF, gray scale of 8 -bit, and the images size is $256 \mathrm{X} 256$ pixels. The analysis programs are performed using MATLAB 6.5 with a PC whose configuration is (Pentium IV, Intel $2.6 \mathrm{GHz}$ processor, 512 Mbytes cash memory, 256 Mbytes RAM).

To study the effect of the implemented restoration techniques on images with atmospheric turbulence blur at three different noise variance. Fig. (3) depicts an example of two original satellite images as shown in Fig. (3.a). Fig. (3.b) shows the noisy degraded image with atmospheric turbulence $\left(\sigma_{G}=1.2\right)$ and noise variance $=0.01$. The results of applying the inverse filter, iterative method, wiener filter, regularized filter and wavelet-based method are shown in Fig. (3.c), Fig. (3.d), Fig. (3.e), Fig. (3.f), and Fig. (3.g) respectively.

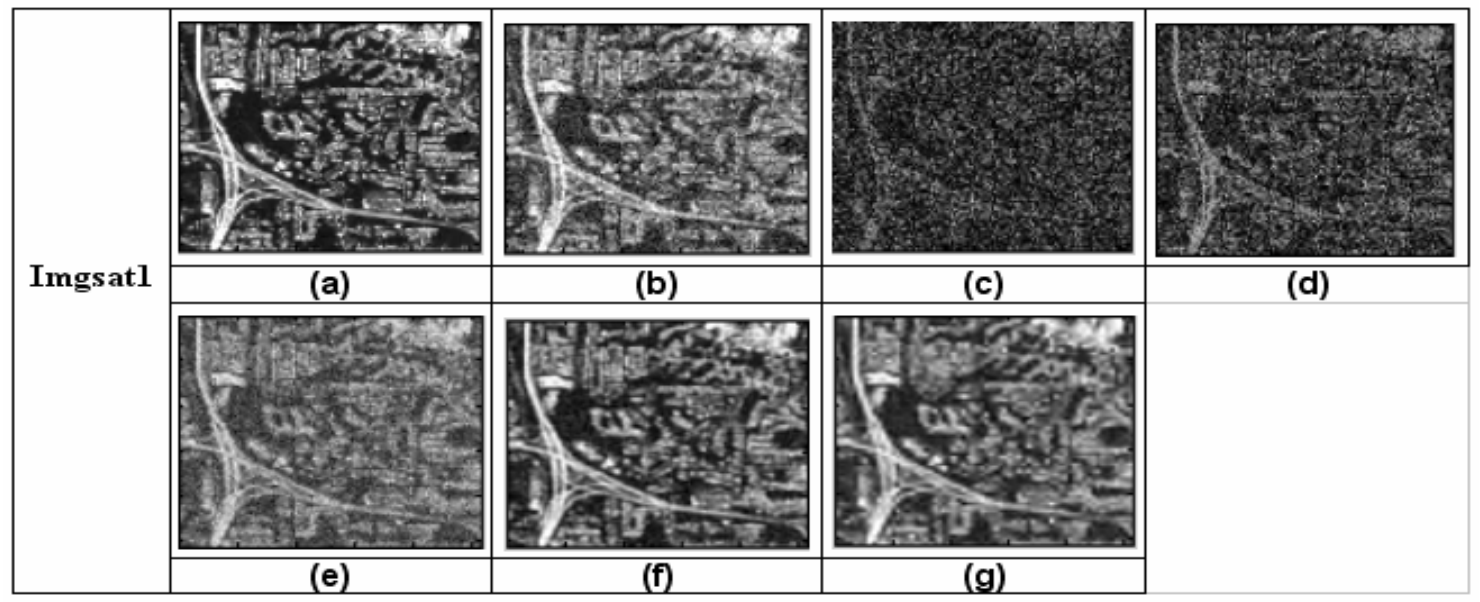


Proceedings of the $5^{\text {th }}$ ICEENG Conference. 16-18 Mav. 2006

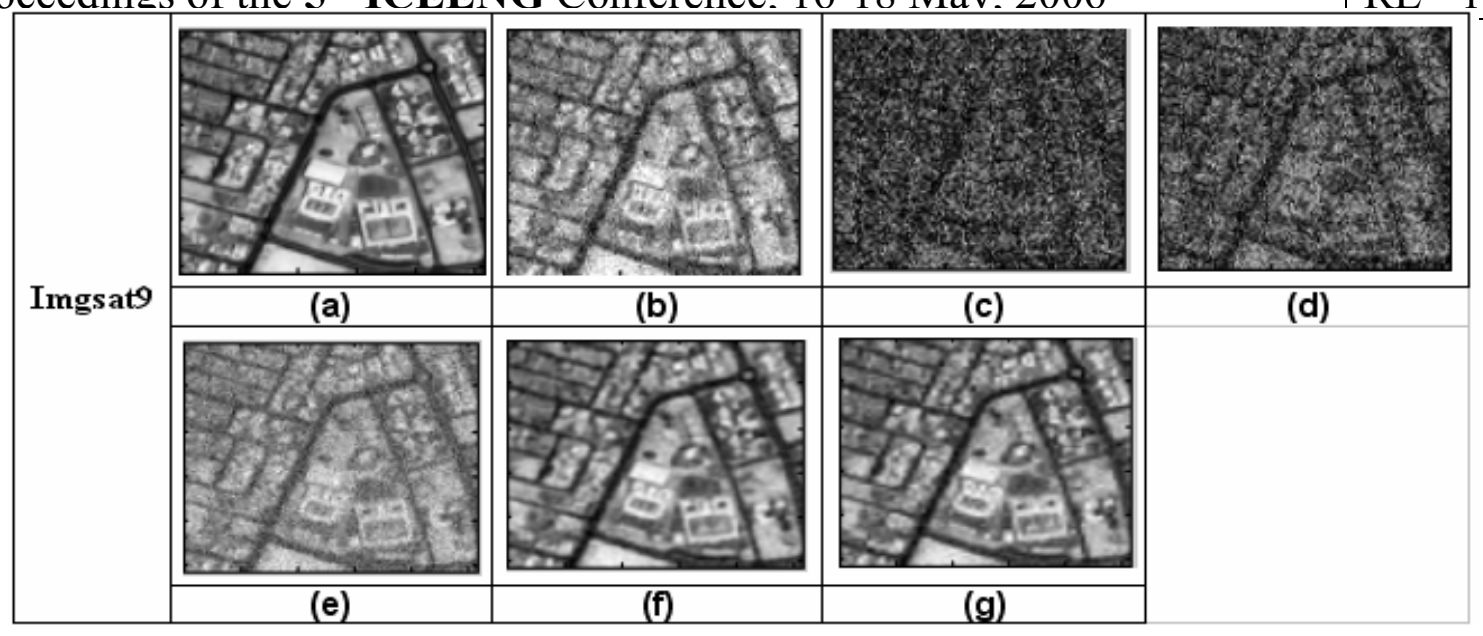

Fig. 3. An example of two satellite images after applying the different image restoration techniques with atmospheric turbulence $\left(\sigma_{G}=1.2\right)$ and noise variance $=0.01$.

To evaluate the performance of the implemented restoration techniques and their capabilities to restore the degraded image as close as possible to the original image. The RMSE and PSNR are computed for 10 satellite sub-images at noise variance $=0.01$, as reported in Table 1. From Table 1, it could be noticed that the wavelet-based method gives high average of PSNR and small average of RMSE with respect to the other restoration techniques.

Table 1. Comparison of the different image restoration techniques with atmospheric turbulence $\left(\sigma_{G}=1.2\right)$ and noise variance $=0.01$.

\begin{tabular}{|c|c|c|c|c|c|c|c|c|c|c|}
\hline \multirow{2}{*}{ Sat Mo } & \multicolumn{2}{|c|}{ Inverse Filter } & \multicolumn{2}{|c|}{ Iterative Method } & \multicolumn{2}{|c|}{ Wiener Filter } & \multicolumn{2}{|c|}{ Regularized Filter } & \multicolumn{2}{|c|}{ Wavelet Method } \\
\hline & PSIIR & RMSE & PSIIR & RMSE & PSIIR & RMSE & PSIIR & RMSE & & \\
\hline Tmgsat 1 & 8.853 & 92.018 & 12.102 & 63.305 & 18.773 & 29.327 & 21.052 & 22.589 & 18.747 & 29.454 \\
\hline & 8.569 & 95.072 & 11.824 & 65.357 & 19.750 & 26.242 & & & & \\
\hline & 8.350 & & 11.3 & & & & & & & 31.296 \\
\hline & 8.720 & 93 & 12.14 & 62.987 & 20.000 & 25.499 & 29. & & 800 & 8.2510 \\
\hline Tmgsat 5 & 10.02 & 80.431 & 13.795 & 52.093 & 20.824 & 23.191 & 22.262 & 19.653 & 5.157 & 14.082 \\
\hline & 9.157 & & 12.6 & & 19.757 & 26. & & & & 11.950 \\
\hline Img & 9.738 & 83.103 & 13.560 & 53.522 & 20.389 & 24.382 & 26.081 & 12.660 & 28.769 & 9.2913 \\
\hline Tmgsat 8 & 9.446 & 85.939 & 12.771 & 58.609 & 19.161 & 28.085 & 19.050 & 28.445 & 20.141 & 25.087 \\
\hline & 8.625 & & & & 19.899 & & & & & \\
\hline Imgsat 10 & 8.892 & 91.606 & 12.441 & 60.876 & 19.857 & 25.923 & 24.438 & 15.297 & 24.595 & 15.023 \\
\hline Average & 9.037 & 90.243 & 12.467 & 60.900 & 19.543 & 27.056 & 24.040 & 17.417 & 24.359 & 17.065 \\
\hline
\end{tabular}

To study the effect of increasing the noise variance on images. Fig. (4) depicts an example of two original satellite images as shown in Fig. (4.a). Fig. (4.b) shows the noisy degraded image with atmospheric turbulence $\left(\sigma_{G}=1.2\right)$ and noise variance $=0.05$. The results of applying the inverse filter, iterative method, wiener filter, regularized filter and wavelet method are shown in Fig. (4.c), Fig. (4.d), Fig. (4.e), Fig. (4.f), and Fig. (4.g) respectively.

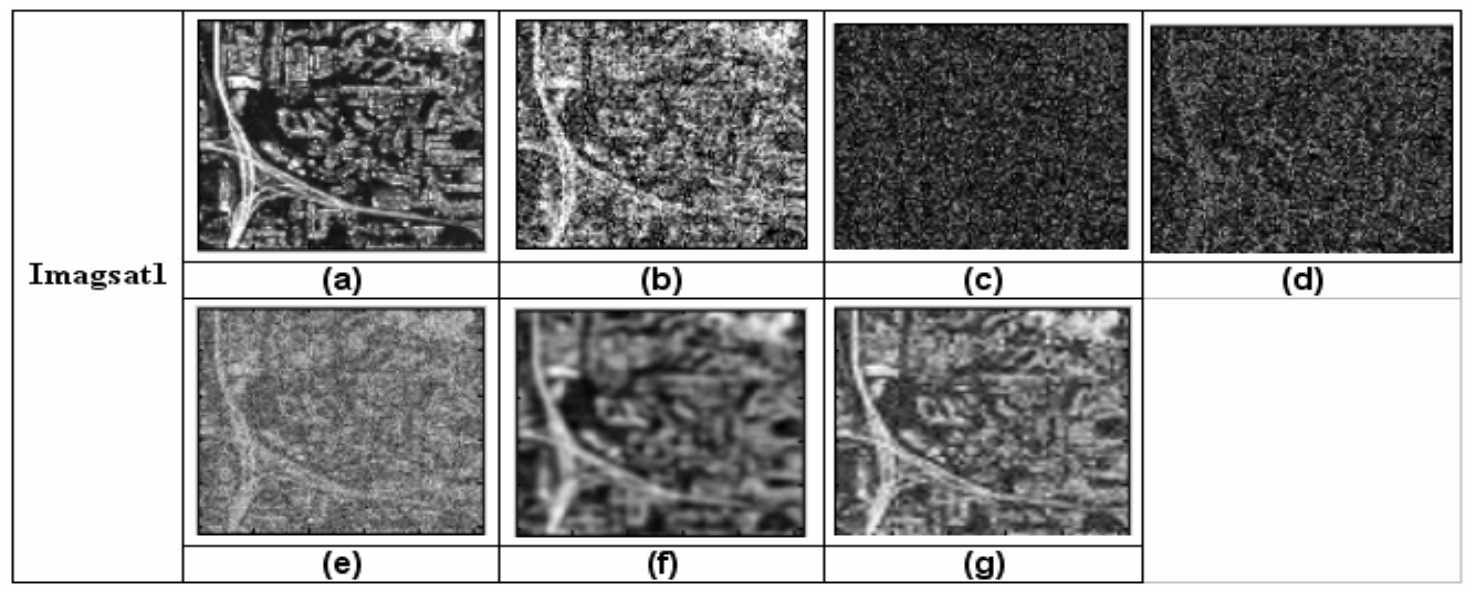




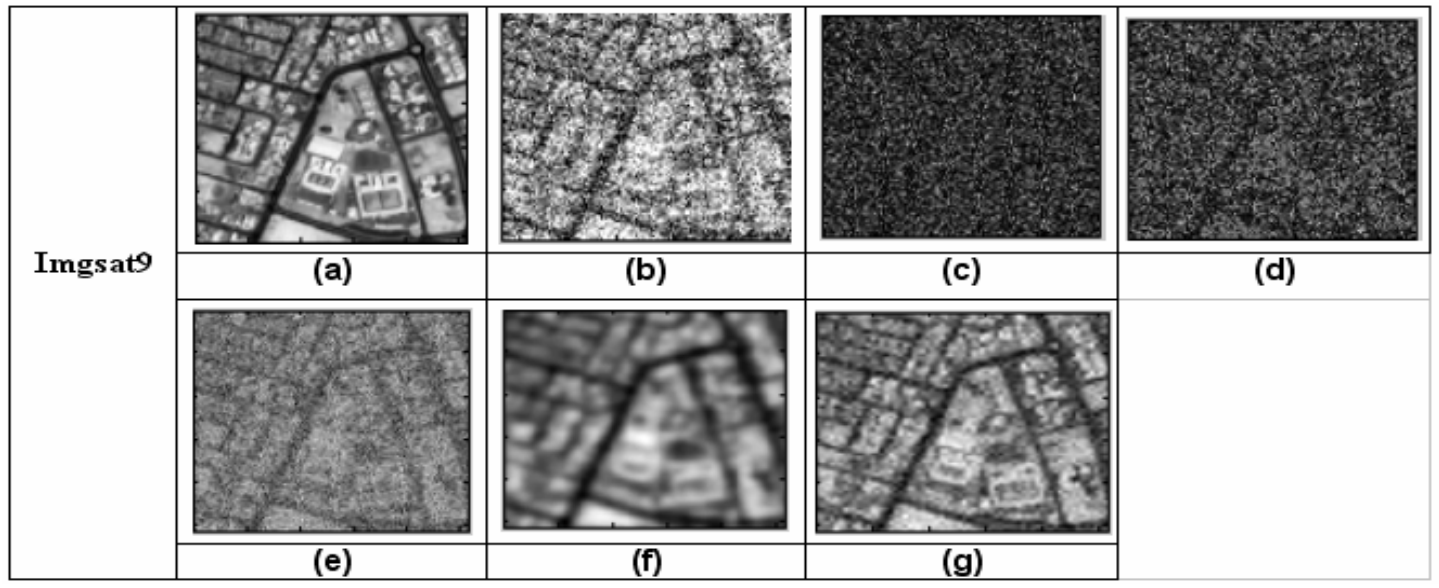

Fig. 4. An example of two satellite images after applying the different image restoration techniques with atmospheric turbulence $\left(\sigma_{G}=1.2\right)$ and noise variance $=0.05$.

Again, The RMSE and PSNR are computed for the same 10 satellite sub-images at noise variance $=0.05$, as reported in Table 2 . From Table 2 , it could be noticed that the waveletbased method gives high average of PSNR and small average of RMSE with respect to the other restoration techniques.

Table 2. Comparison of the different image restoration techniques with atmospheric turbulence $\left(\sigma_{G}=1.2\right)$ and noise variance $=0.05$.

\begin{tabular}{|c|c|c|c|c|c|c|c|c|c|c|}
\hline \multirow{2}{*}{ Sat Ilo } & \multicolumn{2}{|c|}{ Inverse Filter } & \multicolumn{2}{|c|}{ Iterative Method } & \multicolumn{2}{|c|}{ Wiener Filter } & \multicolumn{2}{|c|}{ Regularized Filter } & \multicolumn{2}{|c|}{ Wavelet Method } \\
\hline & PSIIR & RMSE & PSIIR & RMSE & PSIIR & RMSE & PSIIR & RMSE & PSIIR & RMSE \\
\hline Imgsat 1 & 2.878 & 183.063 & 7.167 & 111.735 & 13.337 & 54.913 & 16.764 & 37.008 & 17.884 & \\
\hline Imgsat 2 & 2.806 & 184.599 & 6.989 & 114.037 & 13.244 & 55.501 & 22.991 & 78.070 & 23.194 & 17.652 \\
\hline Tmgsat 3 & 2.921 & 782.165 & 6.888 & 175.378 & 12.759 & 58.688 & 16.328 & 38.914 & 77.546 & 33.824 \\
\hline Tmgsat 4 & 2.808 & 184.554 & 7.094 & 112.670 & 13.397 & 54.536 & 22.780 & 18.515 & 24.736 & 14.780 \\
\hline Tmgsat 5 & 3.613 & 168.207 & 7.971 & 101.847 & 14.533 & 47.851 & 14.737 & 46.736 & 21.202 & 22.203 \\
\hline Tmgsat 6 & 2.835 & 183.976 & 7.353 & 109.361 & 13.522 & 53.756 & 20.340 & 24.518 & 23.414 & 77.211 \\
\hline Tmgsat 7 & 3.101 & 178.431 & 7.743 & 104.559 & 14.031 & 50.694 & 17.112 & 35.555 & 23.596 & 76.854 \\
\hline 8 & 4.445 & 152.855 & 8.069 & 100.711 & 14.586 & 47.554 & 13.281 & 55.270 & 18.276 & 31.098 \\
\hline Tmgsat 9 & 2.984 & T80.854 & 7.187 & T11.478 & 13.539 & 53.649 & 19.895 & 25.809 & 22.642 & 18.811 \\
\hline mgsat 10 & 3.114 & 178.168 & 7.320 & 109.781 & 13.757 & 52.319 & 18.068 & 31.852 & 22.069 & 20.093 \\
\hline Average & 3.150 & 177.690 & 7.378 & 109.160 & 13.670 & 49.089 & 78.229 & 33.224 & 21.455 & 22.506 \\
\hline
\end{tabular}

Fig. (5) depicts an example of two original satellite images as shown in Fig. (5.a). Fig. (5.b) shows the noisy degraded image with atmospheric turbulence $\left(\sigma_{\mathrm{G}}=1.2\right)$ and noise variance $=$ 0.1 . The results of applying the inverse filter, iterative method, wiener filter, regularized filter and wavelet-based method are shown in Fig. (5.c), Fig. (5.d), Fig. (5.e), Fig. (5.f), and Fig. (5.g) respectively.

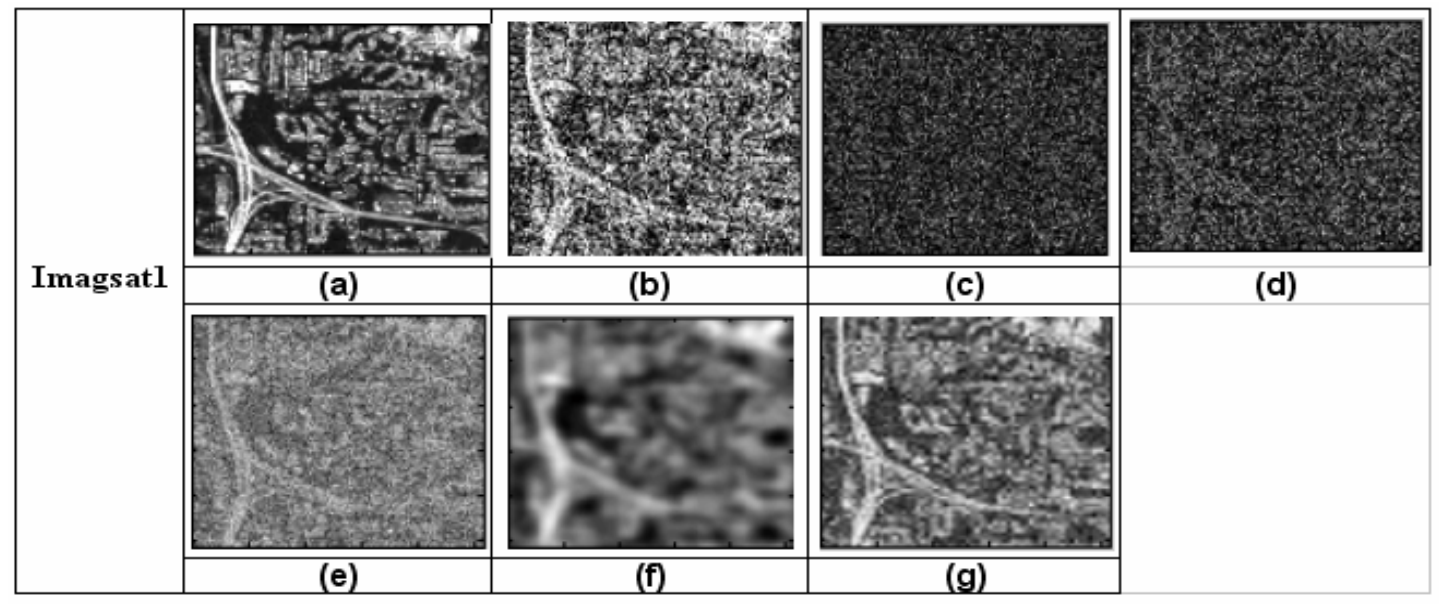




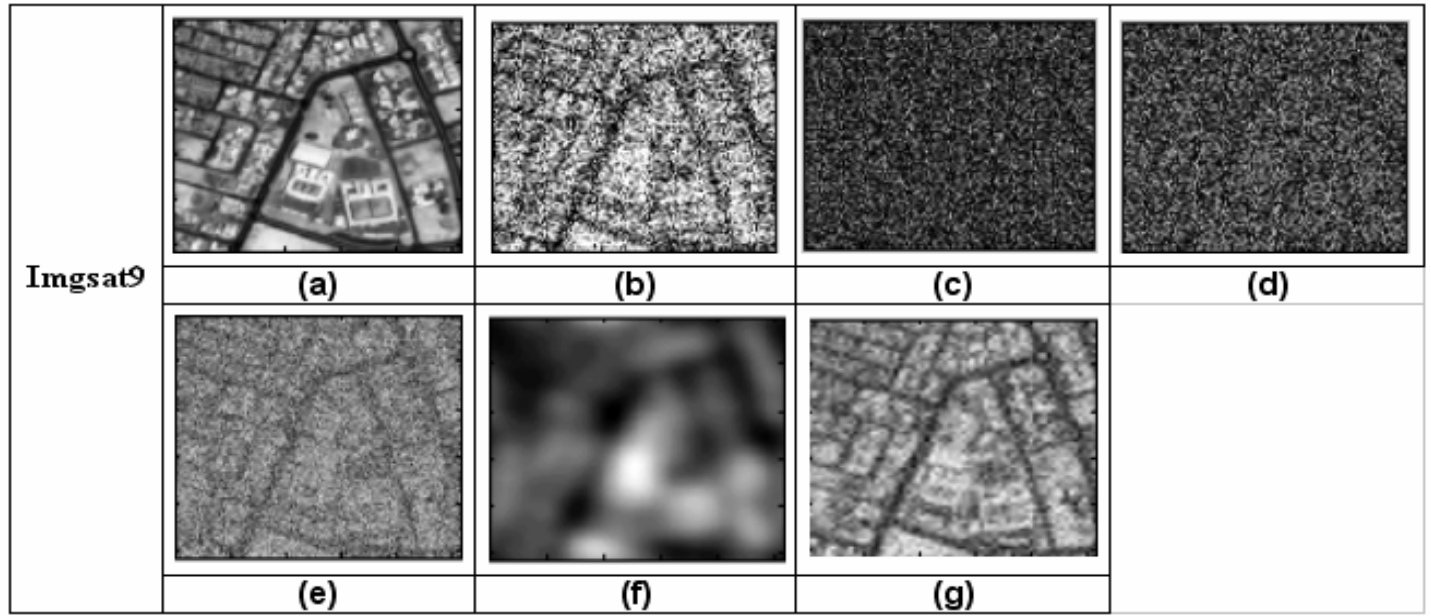

Fig. 5. An example of two satellite images after applying the different image restoration techniques with atmospheric turbulence $\left(\sigma_{G}=1.2\right)$ and noise variance $=0.1$.

Again, The RMSE and PSNR are computed for the same 10 satellite sub-images at noise variance $=0.1$, as reported in Table 3. From Table 3, it could be noticed that the wavelet method gives high average of PSNR and small average of RMSE with respect to the other restoration techniques.

Table 3. Comparison of the different image restoration techniques with atmospheric turbulence $\left(\sigma_{G}=1.2\right)$ and noise variance $=0.1$.

\begin{tabular}{|c|c|c|c|c|c|c|c|c|c|c|}
\hline \multirow{2}{*}{ Sat Ho } & \multicolumn{2}{|c|}{ Inverse Filter } & \multicolumn{2}{|c|}{ Iterative Method } & \multicolumn{2}{|c|}{ Wiener Filter } & \multicolumn{2}{|c|}{ Regularized Filter } & \multicolumn{2}{|c|}{ Wavelet Method } \\
\hline & PSIIR & RMSE & PSIIR & RMSE & PSIIR & RMSE & PSIIR & RMSE & PSIIR & RMSE \\
\hline Tmgsat 1 & 0.492 & 240.945 & 5.145 & 141.722 & 11.117 & 70.901 & 13.460 & 54.141 & 17.204 & 35.180 \\
\hline Tmgsat 2 & 0.425 & 242.809 & 5.153 & 140.882 & 10.978 & 72.044 & 21.452 & 21.574 & 21.678 & 21.020 \\
\hline Tmgst 3 & 0.637 & 236.958 & 5.170 & 140.609 & 10.847 & 73.145 & 12.563 & 60.031 & 76.855 & 36.624 \\
\hline Tmgsat 4 & 0.365 & 244.487 & 5.088 & 741.948 & 11.090 & 71.120 & 18.608 & 29.930 & 22.350 & 19.454 \\
\hline Tmgst 5 & 0.943 & 228.765 & 5.487 & 135.571 & 12.066 & 63.564 & 12.383 & 61.286 & 18.820 & 29.209 \\
\hline Tmgst 6 & 0.326 & 245.594 & 5.075 & 742.155 & 11.242 & 69.893 & 16.752 & 39.710 & 21.615 & 21.172 \\
\hline Imgsat 7 & 0.527 & 239.984 & 5.199 & 140.135 & 11.714 & 66.194 & 16.740 & 37.114 & 20.860 & 23.095 \\
\hline Tmgsat 8 & 2.194 & 798.063 & 6.165 & 125.390 & 12.103 & 63.297 & 9.442 & 85.988 & 16.657 & 37.469 \\
\hline Tmgsat 9 & 0.631 & 237.109 & 5.289 & T38.701 & 11.257 & 69.772 & 14.804 & 46.377 & 20.999 & 22.728 \\
\hline Tmgst 10 & 0.661 & 236.312 & 5.284 & 138.778 & 11.434 & 68.365 & 12.898 & 57.759 & 20.253 & 24.765 \\
\hline Average & 0.720 & 235.100 & 5.305 & 138.590 & 11.384 & 68.829 & 14.850 & 49.391 & 19.729 & 27.071 \\
\hline
\end{tabular}

Fig. (6) depicts the effect of three different noise levels with atmospheric turbulence blur after applying the wavelet restoration technique.

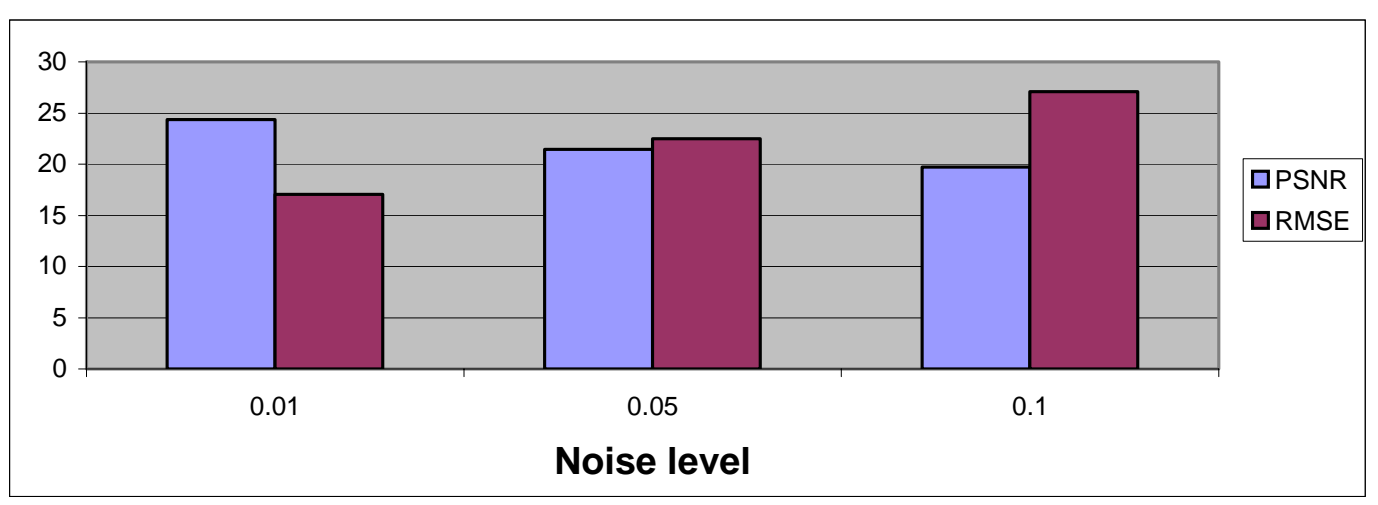

Fig. 6. The effect of increasing the noise levels with atmospheric turbulence blur after applying the wavelet restoration technique. 


\section{6- Conclusion}

The experimental results showed that,

- The inverse filtering and iterative method do not deal well with noise but the iterative method deals a little better with noise than the inverse filtering. Also, the regularized filter requires Knowledge of only the mean and variance of the noise. These parameters usually can be calculated from a given degraded image, so this is an important advantage than the wiener filter. Finally the wavelet-based method is the most suitable restoration technique for satellite images, since it gives high PSNR and small RMSE with respect to the other restoration techniques.

- Increasing the noise level leads to increasing the RMSE and decreasing the PSNR

\section{7- Reference}

[1] Rafel C.Gonzalez, Richard E.Woods, "Digital image processing",.(2000).

[2]... Reginald L. Lagendijk and Jan Biemond,"Handbook of Image and Video Processing".(2000).

[3] J M blackledge, “Digital Image Processing (DIP)".(1986).

[4] H.C. Andrews and B. R. Hunt, "Digital Image Restoration" .(1997).

[5] Internet;Inverse Filtering. http:/www.ownlet.rice.edu/projects/inverse filtering.htm

[6]...J. Biemond, R. L. Lagendijk, and R.M. Mersereau,'Iterative methods for image deblurring,"Proc.IEEE 78, 856-883 (1990).

[7]...A. K. Katsaggelos, "Iterative image restoration algorithm," Opt. Eng. 28, 735-748 (1989).

[8]...R. L. Lagendijk and J. Biemond, Iterative Identification and Restoration of Images (Kluwer, Boston, MA, 1991).

[9]...T. Bretschneider “On the Deconvolution Of Satellite Imegery”.(2002).

[10] B. R. Hunt, "The application of constrained least squares estimation to image restoration by digital computer,'IEEETrans.Comput.2, 805-812 (1997).

[11] A. Jalobeanu, L. Blanc-Féraud , J. Zerubia , “Adaptive parameter estimation for satellite image deconvolution”.(2000).

[12] A. Jalobeanu , L. Blanc-Féraud, J. Zerubia "Satellite image deblurring using complex wavelet packets".(2002).

[13] Internet; Wavelet-based image restoration. http:/www.ownlet.rice.edu/projects/

Wavelet-based image restoration.htm. 